\title{
Interaction induced deformation in momentum distribution of spin polarized nuclear matter
}

\author{
T. Frick, H. Müther, A. Sedrakian \\ Institut für Theoretische Physik, Universität Tübingen, D-72076 Tübingen, Germany \\ Effects of spin polarization on the structure of symmetric nuclear matter and \\ pure neutron matter are investigated. We show that the spin polarization \\ induces a deformation of the Fermi spheres for nucleons with spin parallel \\ and opposite to the polarization axes. This feature can be related to the \\ structure of the one pion exchange contribution to a realistic nucleon-nucleon \\ interaction. While the anisotropies in the momentum distribution lower the \\ energy of the system by small amount, the associated variations of the single \\ particle energies with the angle between the polarization axis and the particle \\ momentum are significant.
}

The magnetic susceptibility of nuclear matter has received a considerable amount of attention as this quantity is relevant for the studies in astrophysics of compact objects. The strong magnetic fields in the interiors of neutron stars may lead to a magnetization of the material of nuclear matter, which implies a spin polarization of the interacting nucleons. Various groups have discussed the possibility of a phase transition of normal neutron matter to a ferromagnetic state [1]. With realistic models of the nucleon-nucleon (NN) interaction, however, such a phase transition seems to be suppressed for neutron matter up to densities well above the saturation density $\rho_{0}$ of symmetric nuclear matter. Moreover, it turns out that the NN interaction even tends to reduce the susceptibility below the value of a noninteracting Fermi gas

$$
\chi_{F}=\frac{\mu^{2} m k_{F}}{\hbar^{2} \pi^{2}}
$$

where $\mu$ and $m$ represent the magnetic moment and the mass of the neutron while $k_{F}$ stands for the Fermi momentum of system. This reduction is related to the density of states at the Fermi surface, which can be expressed in terms of an effective mass $m^{*}$, which is typically smaller than the bare nucleon mass $m$, and the strength of the spin-spin interaction, represented by a Landau parameter $G_{0}$. An estimate for the susceptibility is then

$$
\frac{\chi}{\chi_{F}} \approx \frac{m^{*}}{m\left(1+G_{0}\right)}
$$

If, however, the spin-spin interaction becomes attractive at densities above the nuclear saturation density, as it is the case for some effective interactions like the Skyrme force, the system may undergo a phase transition to a ferromagnetic state [8,9].

The polarizability of nuclear matter or its spin-spin correlation function is also an important ingredient for the study of the interaction of neutrinos with nuclear matter. This interaction of neutrinos with dense matter is of high importance for the simulation of supernova explosion and the cooling of neutron stars. In order to compute the mean free path of neutrinos 
in nuclear matter one has to determine the nuclear response function for density and spin excitations [9 12] in particular for lower energies. These response functions are essentially characterized by the single-particle spectrum of the nucleons with momenta $p$ around the Fermi momentum, which is often parameterized in terms of an effective mass and momentum independent single-particle potential $U$

$$
\varepsilon(p)=\frac{p^{2}}{2 m^{*}}+U
$$

and the residual interaction, which is conveniently characterized by the Landau parameters $F_{0}$ and $G_{0}$ for the density and spin response functions, respectively.

The studies of the nuclear polarizability and the nuclear response functions all consider a conventional model for nuclear matter, where the single-particle states are occupied according to the magnitude of the single-particle momentum $p$. As a typical example we refer to the Brueckner-Hartree-Fock (BHF) calculation of Vidaña et al. [7], who evaluate the single-particle energies for nucleons with momentum $p$ and spin projection $s$ according to

$$
\varepsilon(p, s)=\frac{p^{2}}{2 m}+\sum_{s^{\prime}} \int \frac{d^{3} q}{(2 \pi \hbar)^{3}}\left\langle p s, q s^{\prime}\right| G\left(\omega=\varepsilon(p, s)+\varepsilon\left(q, s^{\prime}\right)\left|p s, q s^{\prime}\right\rangle_{A} n\left(q, s^{\prime}\right),\right.
$$

where $G$-matrix is the solution of the Bethe-Goldstone equation for a realistic model of the $\mathrm{NN}$ interaction, $\omega$ is the starting energy and $n\left(q, s^{\prime}\right)$ is the occupation probability of nucleons with momentum $q$ and spin projection $s^{\prime}$. In the BHF approximation for systems at temperature $T=0$ this occupation probability is represented by a step function which restricts the occupation to states with momenta below the Fermi momentum $k_{F}\left(s^{\prime}\right)$ of nucleons with spin projection $s^{\prime}$. Note that in order to shorten the notation, we have suppressed all labels referring to the isospin of the nucleons.

It is well known that a realistic NN interaction contains strong tensor and spin-orbit components, which couple the spin and momentum or spatial dependence of the interaction. In the case of the deuteron these tensor components lead to dumbbell shape if one considers deuterons with spin projection $M_{s}= \pm 1$ [13]. These considerations lead to the conjecture that also the momentum distribution of polarized nuclear matter could be deformed with respect to the axis of spin polarization. Configurations with deformed momentum distributions could be lower in energy than the spherical ones discussed so far, which would affect the polarizability. A deformed momentum distribution would also be connected with the single-particle spectrum, in which the single-particle energies depend on the magnitude and the direction of the momentum $\vec{p}$ of the nucleon under consideration. This would lead to different response functions and influence the mean free path of neutrinos as well as other properties of the nuclear matter.

In order to investigate this question, we consider a Fermi momentum that depends on the angle $\vartheta$ with respect to the spin-polarization axis, parameterized in terms of

$$
k_{F}(s, \vartheta)=k_{F 0}(s)\left(1+\delta(s) \cos ^{2} \vartheta\right),
$$

a parameterization which turned out to be useful. With this assumption we can calculate the contribution of the single-particle potential $U(\vec{p}, s)$ to the single-particle energy in (四), 
employing the partial wave expansion for the $G$-matrix as outlined e.g. by Haftel and Tabakin 14

$$
\begin{aligned}
U(\vec{p}, s)= & \sum_{s^{\prime}, \mathcal{S}, L^{\prime}, L, J, M_{L}, M} \int \frac{d^{3} q}{(2 \pi \hbar)^{3}} n\left(\vec{q}, s^{\prime}\right)\left(L^{\prime} M_{L}, \mathcal{S} s+s^{\prime} \mid J M\right)\left(L M_{L}, \mathcal{S} s+s^{\prime} \mid J M\right) \\
& \times Y_{L^{\prime} M_{L}}^{*}\left(\hat{k}_{r}\right) Y_{L M_{L}}\left(\hat{k}_{r}\right) i^{\left(L^{\prime}-L\right)} G_{L^{\prime} L}^{J \mathcal{S}}\left(\omega, K_{C M}, k_{r}\right)
\end{aligned}
$$

with $\vec{K}_{c m}$ and $\vec{k}_{r}$ referring to the center of mass and relative momentum of the interacting pair of nucleons, respectively. The brackets $\left(L m_{L}, \mathcal{S}, M_{S} \mid J M\right)$ denote the Clebsch-Gordan coefficient for the coupling of the spin and orbital angular momentum of the relative motion. Note that for a system without spin-polarization the spherical harmonics $Y_{L, M}$ drop out. If, however, we assume a spin-polarization Eq. (6) yields a single-particle potential which depends not only on the magnitude of the momentum $\vec{p}$ but also on the angle relative to the axis of spin-polarization, even if we assume spherical momentum distributions $n\left(\vec{q}, s^{\prime}\right)$, i.e. $\delta\left(s^{\prime}\right)=0$ in (5). From the momentum dependence of the resulting single-particle energies, one can find a Fermi momentum according to (5) and recalculate the single-particle energy with this deformed momentum distribution until a self-consistent solution is obtained.

Results for the single-particle potential of such BHF calculations are displayed in Fig. 1 for nuclear matter at the empirical saturation density $\rho_{0}$ with a spin polarization

$$
\Delta \rho=\frac{\rho^{\uparrow}-\rho^{\downarrow}}{\rho_{0}}
$$

of 25 percent. The calculation have been performed using the charge-dependent CDBonn NN interaction of Machleidt et al. [15]. The spin asymmetry as well as the angle-dependence of the single-particle spectrum have been ignored in the Pauli operator and starting energy, which were used to solve the Bethe-Goldstone equation. The single-particle potential for the nucleons with spin parallel to the polarization axis (left panel of Fig. 1) and a momentum of $1.46 \mathrm{fm}^{-1}$ (the Fermi momentum of the spin up momentum distribution if it would be spherical) is about $2.7 \mathrm{MeV}$ more attractive in the direction of the momentum perpendicular to the polarization axis $(\theta=\pi / 2)$ than for a momentum parallel or antiparallel to this axis. This means that we obtain an oblate momentum distribution for the nucleons with spin parallel to the polarization axis, which can be parameterized according to Eq. (5) with a deformation parameter $\delta(\uparrow) \approx-0.03$. It is worth noting that this oblate deformation is in line with the observation made for the deuteron [13 as the dumbbell shape of the density distribution mentioned above corresponds to a momentum distribution with oblate deformation.

The situation is opposite for the nucleons with spin antiparallel to the polarization axis. In this case we observe a smaller single-particle energy for momenta parallel to the polarization axis as compared to the momentum perpendicular to this axis (see right panel of Fig. 1). This leads to a prolate deformation of the momentum distribution of the nucleon with spin antiparallel to the polarization axis.

The angle-dependence of the single-particle potential is of course much stronger in the case of totally polarized nuclear matter. In this case we obtain a energy difference of around $20 \mathrm{MeV}$ between the single-particle energies for the direction of the momentum parallel as 
compared to perpendicular to the polarization axis, if we consider a momentum of 1.72 $\mathrm{fm}^{-1}$, which is the Fermi momentum of the spherical distribution for this polarization. This corresponds to a deformation parameter $\delta(\uparrow) \approx-0.15$ in the parameterization of Eq. (5).

The effects are weaker for pure neutron matter. For example, in the case of completely polarized neutron matter at density $\rho_{0}$, the gain in the single-particle energy is around $6 \mathrm{MeV}$ if the momentum is perpendicular to the polarization axis $z$ as compared to the same momentum parallel to $z$ (assuming a neutron with spin parallel to $z$ and a momentum $p=2.16 \mathrm{fm}^{-1}$ which is the Fermi momentum of the spherical distribution). This corresponds to a deformation parameter $\delta(\uparrow) \approx-0.03$.

To understand the origin of this deformation we recall that within the Hartree-Fock approximation the non-locality or momentum dependence of the self-energy of a nucleon in nuclear matter is mainly due to the Fock-exchange contribution in (4). For the sake of simplicity let us consider completely polarized nuclear matter. In this case the contribution of the one-pion exchange to the Fock term in Eq. (4) for a nucleon with spin parallel to the polarization axis can be described by

$$
\Delta U_{\pi}(\vec{p}, \uparrow) \sim \int d^{3} q\left\langle\vec{p} \uparrow, \vec{q} \uparrow\left|\frac{\left(\vec{\sigma}_{1} \cdot \vec{k}\right)\left(\vec{\sigma}_{2} \cdot \vec{k}\right)}{m_{\pi}^{2}+k^{2}}\right| \vec{q} \uparrow, \vec{p} \uparrow\right\rangle n(\vec{q}, \uparrow)
$$

In this equation we have approximated the one-pion exchange term by its static, nonrelativistic reduction in which $\vec{\sigma}_{i}$ represents the vector of Pauli matrices acting on nucleon $i$ and $\vec{k}$ denotes the momentum transfer which is given in terms of the momenta of the interacting nucleons by $\vec{k}=\vec{p}-\vec{q}$. Since the spin part of the matrix element in (8) yields results different from zero only for the $z$-component of the spin matrices $(z$-axis parallel to the polarization axis) one obtains

$$
\Delta U_{\pi}(\vec{p}, \uparrow) \sim \int d^{3} q \frac{\left(p_{z}-q_{z}\right)^{2}}{m_{\pi}^{2}+k^{2}} n(\vec{q}, \uparrow) .
$$

Indeed, we find that the self-energy for completely spin-polarized nuclear matter increases with the absolute value of $p_{z}$, but is rather weakly dependent on $p_{x}$ and $p_{y}$.

The situation is different, if we consider the self-energy of a nucleon with spin antiparallel to the axis of spin polarization. In this case we have to consider matrix elements of the form

$$
\int d^{3} q\left\langle\vec{p} \downarrow, \vec{q} \uparrow\left|\frac{\left(\vec{\sigma}_{1} \cdot \vec{k}\right)\left(\vec{\sigma}_{2} \cdot \vec{k}\right)}{m_{\pi}^{2}+k^{2}}\right| \vec{q} \uparrow, \vec{p} \downarrow\right\rangle n(\vec{q}, \uparrow),
$$

which lead to a self-energy which increases with the component of $\vec{p}$ perpendicular to the $z$-axis. This means that the angle-dependence of the self-energy can be understood qualitatively in terms of the Fock contribution of the pion-exchange part of the NN interaction.

We will turn next to the effect of the deformed momentum distribution on the total energy. The energy per nucleon is given by

$$
\frac{E}{A}=\frac{\sum_{s} \int d^{3} p n(\vec{p}, s) \frac{1}{2}\left(\frac{p^{2}}{2 m}+\varepsilon(\vec{p}, s)\right)}{\sum_{s} \int d^{3} p n(\vec{p}, s)} .
$$


One finds that the gain in energy due to the deformation of the momentum distribution is rather small (see table (1). Even in the case of completely polarized nuclear matter, the deformed momentum distribution yields an energy per nucleon which is $0.53 \mathrm{MeV}$ smaller than the energy calculated for the spherical momentum distribution. This is a rather small correction compared to the energy difference between the polarized and unpolarized energy. Therefore we cannot expect that the effects of deformed momentum distributions will alter the conclusions of e.g. [7] about the equation of state for spin polarized matter or the possible existence of a ferromagnetic state of nuclear or neutron matter. The situation, however, might be compared to the deformation degrees of freedom in finite nuclei. The possibility to form deformed nuclei is not very relevant for the total energy of finite nuclei. The gain in energy due to deformation of nuclei is typically of the order of $0.05 \mathrm{MeV}$ per nucleon or even less (see e.g. [16]). The deformation, however, has rather significant effects on the excitation spectrum at low energies, which is quite different for spherical as compared to deformed nuclei. Also, the dependence of the single-particle energies on the direction of the nucleon momentum in spin polarized nuclear matter may lead to quite different response functions at low excitation energies. This may have a significant effect e.g. on the neutrino propagation in neutron stars with large magnetic fields.

We like to acknowledge the financial support by the Europäische Graduiertenkolleg Tübingen - Basel (DFG - SNF) and the Sonderforschungsbereich 382 (DFG).

[1] S.D. Silverstein, Phys. Rev. Lett. 23, 139 (1969) .

[2] M.J. Rice, Phys. Lett. 29 A, 637 (1969) .

[3] E. Østgaard, Nucl. Phys. A 154, 202 (1970) .

[4] J.W. Clark, Phys. Rev. Lett. 23, 1463 (1969) .

[5] V.R. Pandharipande, V.K. Garde, and J.K. Srivastava, Phys. Lett. 38 B, 485 (1972) .

[6] S. Fantoni, A. Saarsa, and K.E. Schmidt, preprint nucl-th/0106026.

[7] I. Vidaña, A. Polls, and A. Ramos, preprint nucl-th/0111055.

[8] A. Vidaurre, J. Navarro, and J. Bernabéu, Astron. Astrophys 135, 361 (1984) .

[9] S. Reddy, M. Prakash, J.M. Lattimer, and J.A. Pons, Phys. Rev. C 59, 2888 (1999) .

[10] R.F. Sawyer, Phys. Rev. C 40, 865 (1989) .

[11] J. Navarro, E.S. Hernandez, and D. Vautherin, Phys. Rev. C 60, 045801 (1999) .

[12] E.N.E. van Dalen, A.E.L. Dieperink, A. Sedrakian, and R.G.E. Timmermans, Astron. Astrophys 360, 549 (2000) .

[13] J.L. Forest, V.R. Pandharipande, S.C. Pieper, R.B. Wiringa, R. Schiavilla, and A. Arriaga, Phys. Rev. C 54, 646 (1996) .

[14] M.I. Haftel and F. Tabakin, Nucl. Phys. A 158, 1 (1970) .

[15] R. Machleidt, F. Sammarruca, and Y. Song, Phys. Rev. C 53, R1483 (1996) ; R. Machleidt, Phys. Rev. C 63, 024001 (2001) .

[16] H. Müther, K. Goeke, K. Allaart, and A. Faessler, Phys. Rev. C 53, 1467 (1977) . 


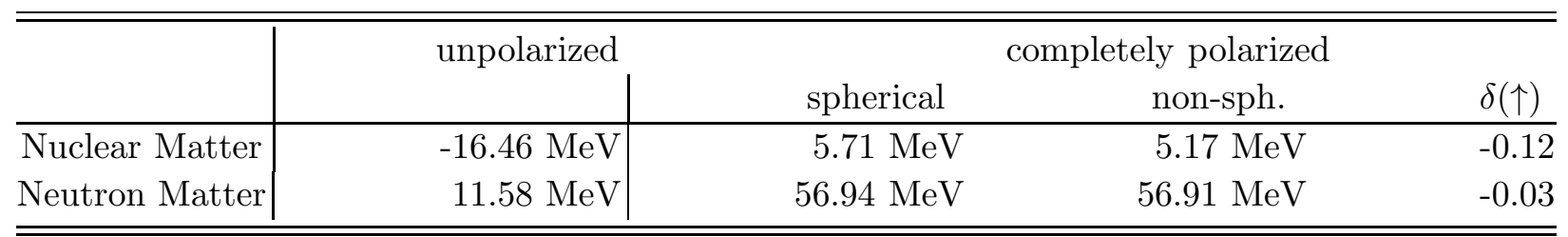

TABLE I. Energy per nucleon for nuclear matter and neutron matter of density $\rho_{0}=0.17 \mathrm{fm}^{-3}$. Results are presented for the unpolarized system and the system with complete spin polarization. For this case we show the energies assuming a spherical and non-spherical momentum distribution. The last column shows the deformation parameter for the self-consistent solution according to Eq. (5)

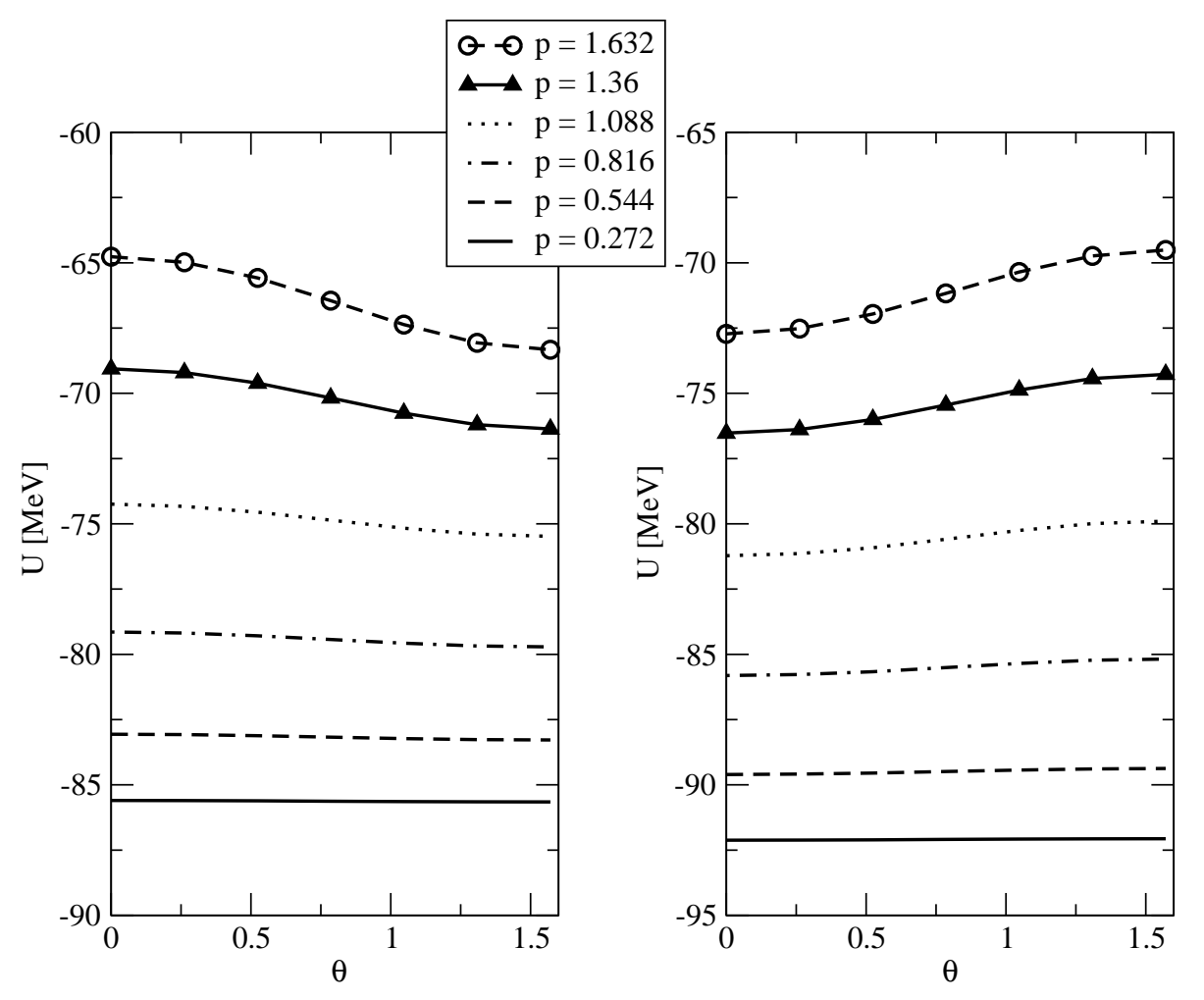

FIG. 1. Single-particle potentials for nucleons with s pin parallel (left panel) and antiparallel (right panel) to the spin polarization axis as a function of the angle $\theta$ between their momentum and the polarization axis in nuclear matter. Results are given for various values for the modulus of the momentum $p$ (expressed in $\mathrm{fm}^{-1}$ as indicated in the legend). The single-particle potentials are symmetric around $\theta=\pi / 2$. 